\title{
Effect of Aerosol Devices and Administration Techniques on Drug Delivery in a Simulated Spontaneously Breathing Pediatric Tracheostomy Model
}

\author{
Bshayer R Alhamad MSc, James B Fink PhD RRT FAARC, \\ Robert J Harwood MSA RRT FAARC, Meryl M Sheard MSc RRT, and \\ Arzu Ari PhD RRT PT CPFT FAARC
}

\begin{abstract}
BACKGROUND: This study was conducted to compare the efficiency of jet nebulizers, vibrating mesh nebulizers, and pressurized metered-dose inhalers (pMDI) during assisted and unassisted administration techniques using a simulated spontaneously breathing pediatric model with a tracheostomy tube (TT). METHODS: An in vitro breathing model consisting of an uncuffed TT (4.5-mm inner diameter) was attached to a collecting filter (Respirgard) connected to a dualchamber test lung and a ventilator (Hamilton Medical) to simulate breathing parameters of a 2-y-old child (breathing frequency, 25 breaths/min; tidal volume, $150 \mathrm{~mL}$; inspiratory time, $0.8 \mathrm{~s}$; peak inspiratory flow, $20 \mathrm{~L} / \mathrm{min}$ ). Albuterol sulfate was administered using a jet nebulizer (MicroMist, $2.5 \mathrm{mg} / 3 \mathrm{~mL}$ ), vibrating mesh nebulizer (Aeroneb Solo, $2.5 \mathrm{mg} / 3 \mathrm{~mL}$ ), and pMDI (ProAir HFA, $432 \mu \mathrm{g}$ ). Each device was tested 5 times with an unassisted technique (direct administration of aerosols with simulated spontaneous breathing) and with an assisted technique (using a manual resuscitation bag in conjunction with an aerosol device and synchronized with inspiration). Drug collected on the filter was analyzed by spectrophotometry. RESULTS: With the unassisted technique, the pMDI had the highest inhaled mass percent (IM\%, $47.15 \pm 7.82 \%$ ), followed by the vibrating mesh nebulizer $(19.77 \pm 2.99 \%)$ and the jet nebulizer $(5.88 \pm 0.77 \%, P=.002)$. IM was greater with the vibrating mesh nebulizer $(0.49 \pm .07 \mathrm{mg})$ than with the pMDI $(0.20 \pm 0.03 \mathrm{mg})$ and the jet nebulizer $(0.15 \pm 0.01 \mathrm{mg}, P=.007)$. The trend of lower deposition with the assisted versus unassisted technique was not significant for the jet nebulizer $(P=.46)$, vibrating mesh nebulizer $(P=.19)$, and pMDI $(P=.64)$. CONCLUSIONS: In this in vitro pediatric breathing model with a TT, the pMDI delivered the highest IM\%, whereas the vibrating mesh nebulizer delivered the highest IM. The jet nebulizer was the least efficient device. Delivery efficiency was similar with unassisted and assisted administration techniques. Key words: aerosols; drug delivery; nebulizers; metered dose inhaler; albuterol; pediatrics; tracheostomy. [Respir Care 2015;60(7):1026-1032. (C) 2015 Daedalus Enterprises]
\end{abstract}

The authors are affiliated with the Division of Respiratory Therapy, Georgia State University, Atlanta, Georgia.

Ms Alhamad presented a version of this paper at the 19th Congress of the International Society for Aerosol in Medicine, held April 6-10, 2013, in Chapel Hill, North Carolina.

Dr Fink is an adjunct faculty member at Georgia State University and participated in this study in that role. Dr Fink's primary employer is his consulting business, James B Fink LLC, which serves the biotech indus- try, whose clients include Aerogen, Dance Biopharm, Parion, Bayer, and Aridis. Dr Ari had an unrestricted grant from Aerogen in 2012. The other authors have disclosed no conflicts of interest.

Correspondence: Arzu Ari PhD RRT PT CPFT FAARC, Division of Respiratory Therapy, Byrdine F Lewis School of Nursing and Health Professions, Georgia State University, PO Box 4019, Atlanta, GA 303024019. E-mail: arzuari@ hotmail.com.

DOI: $10.4187 /$ respcare. 03592 


\section{Introduction}

Aerosol therapy plays an integral role in the treatment of pediatric respiratory diseases. Although multiple studies have focused on aerosol delivery to mechanically ventilated subjects via an endotracheal tube (ETT), ${ }^{1-4}$ little is known about aerosol delivery to spontaneously breathing tracheostomized patients, ${ }^{5,6}$ particularly children.7,8 Inhaled aerosol agents, such as bronchodilators, corticosteroids, antibiotics, and mucolytics, are commonly delivered to spontaneously breathing pediatric patients with a tracheostomy. Administering therapeutic inhaled aerosols to pediatric patients is a challenge. The pediatric population has different ranges of age groups, resulting in patients with different airway sizes and breathing patterns. ${ }^{9}$ Young children have smaller airway sizes, lower tidal volumes $\left(\mathrm{V}_{\mathrm{T}}\right)$, and higher breathing frequency compared with older children. ${ }^{9}$ These patient-related factors impact the deposition of aerosol drugs in the lungs. ${ }^{9,10}$ The presence of an artificial airway, such as a tracheostomy tube (TT) or an ETT, influences the deposition of inhaled aerosols in the lungs. ${ }^{11}$ Therefore, it is important to select an efficient aerosol delivery device and the proper administration technique to enhance aerosol delivery in pediatric patients with a tracheostomy since the effectiveness of aerosol therapy is largely device- and technique-dependent.

Despite the widespread use of aerosol therapy in children with a tracheostomy, little empirical evidence is available in the literature, ${ }^{7,8}$ and there is no clinical guideline for administration of aerosols to this patient population. ${ }^{12}$ A previous survey revealed a wide variation in the practice of aerosol drug delivery in spontaneously breathing pediatric subjects with a tracheostomy. ${ }^{13}$ Therefore, in this study, we simulated inhaled drug delivery to spontaneously breathing tracheostomized pediatric subjects with the aim of providing guidance to clinicians to achieve appropriate target dosing to the lower airways with the range of aerosol generators commonly in clinical use. Previous reports of models of spontaneously breathing children with a tracheostomy have been limited to pressurized metered-dose inhalers (pMDIs) with various adapters and comparison of different pneumatic nebulizers, such as simple jet, breath-enhanced, and breath-actuated nebulizers. ${ }^{7,8}$ Direct comparison of pMDIs, jet nebulizers, and vibrating mesh nebulizers regarding aerosol delivery in this patient population has not been reported. Therefore, in this study, we assessed the efficiency of these aerosol devices in drug delivery distal to the TT in a simulated spontaneously breathing pediatric model with a tracheostomy. A jet nebulizer and pMDI were tested in this study because they have long been considered the most common means of providing therapeutic inhaled aerosols to pediatric patients with a tracheostomy. ${ }^{13}$ Because information about the efficiency of a vibrating mesh nebulizer in aerosol delivery

\section{QUICK LOOK}

\section{Current knowledge}

Aerosol therapy plays an integral role in the treatment of pediatric respiratory diseases. Although multiple studies have focused on aerosol delivery to mechanically ventilated subjects, little is known about aerosol delivery to spontaneously breathing tracheostomized children.

\section{What this paper contributes to our knowledge}

Drug delivered distal to the tracheostomy tube was influenced by the type of aerosol device used in this pediatric model. The metered-dose inhaler was the most efficient in terms of inhaled mass percent, whereas the vibrating mesh nebulizer was the most efficient in terms of inhaled mass due to the larger nominal dose. The jet nebulizer was the least efficient aerosol device.

in spontaneously breathing pediatric patients with TTs is still lacking, a vibrating mesh nebulizer was included in this study to quantify its aerosol deposition and to compare it with the pMDI and jet nebulizer.

Jet nebulizers, pMDIs, and vibrating mesh nebulizers can be used either alone (unassisted technique) or in conjunction with a manual resuscitation bag (assisted technique). Although previous studies reported that the assisted technique enhances aerosol deposition, ${ }^{8,11}$ additional research comparing assisted and unassisted administrative techniques in aerosol delivery to patients with a tracheostomy is warranted. Therefore, the objectives of this study were to identify the most effective device options for administering inhaled bronchodilators via a pediatric TT and to determine the best technique to administer inhaled aerosols via a pediatric TT (assisted or unassisted technique).

\section{Methods}

\section{Simulated Spontaneously Breathing Model}

The model simulating the spontaneous breathing of a 2-y-old child with a TT is shown in Figure 1. The aerosol device is interfaced to the inlet of an uncuffed TT (Shiley, Covidien, Mansfield, Massachusetts) with an inner diameter of $4.5 \mathrm{~mm}^{14}$ and a collecting filter (Respirgard-II 303, Vital Signs, Brooklyn, New York) through a protecting filter to the test chamber of a dual-chamber test lung (TTL/PneuView system, adult/infant lung simulator, Michigan Instruments, Grand Rapids, Michigan). As the ventilator (Hamilton Medical, Reno, Nevada) cycles, the trigger chamber fills, and the lift bar raises the test cham- 


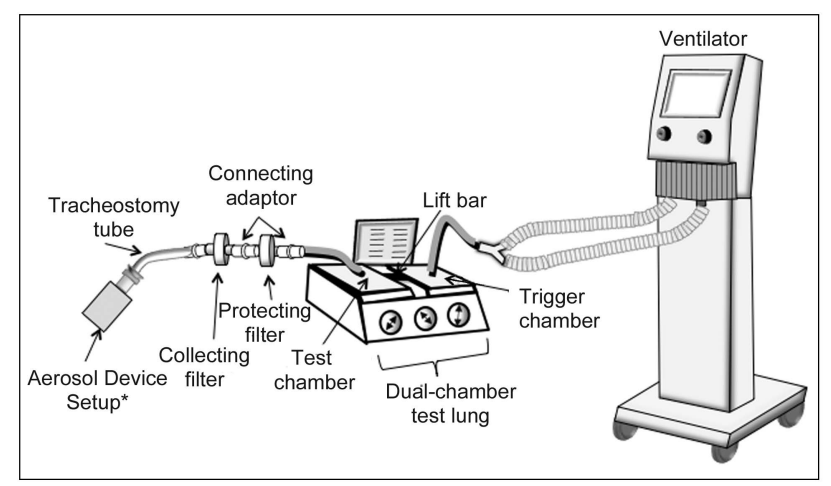

Fig. 1. Primary experimental setup for both assisted and unassisted techniques with each aerosol device used. Each aerosol device setup is attached to a tracheostomy tube with a collecting filter distal to the tube and a secondary protecting filter to the test chamber of a dual-chamber test lung, with a lift bar connected to a trigger chamber, which is connected to a ventilator. As the ventilator applies gas to the trigger chamber, the lift bar raises the test chamber to simulate spontaneous inspiration. ${ }^{*}=$ Aerosol device setup for the jet nebulizer, vibrating mesh nebulizer, and pressurized metered-dose inhaler as detailed in Figure 2.

ber, simulating inspiration. ${ }^{5,15}$ Expiration of the test chamber is passive based on the compliance and resistance set on the chamber. Breathing parameters were set to simulate a breathing frequency of 25 breaths $/ \mathrm{min}$, a $\mathrm{V}_{\mathrm{T}}$ of $150 \mathrm{~mL}$, an inspiratory time of $0.8 \mathrm{~s}$, and a peak inspiratory flow of $20 \mathrm{~L} / \mathrm{min}$ through the TT, as suitable for a 2 -y-old child. ${ }^{16-18}$ The same simulated spontaneously breathing model and parameters were used for both assisted and unassisted techniques.

\section{Types and Operation of Aerosol Devices}

Three types of aerosol devices were tested in this study: (1) a jet nebulizer (Micro Mist, Hudson RCI/Teleflex Medical, Research Triangle Park, North Carolina), (2) a vibrating mesh nebulizer (Aeroneb Solo, Aerogen, Mountain View, California), and (3) a pMDI (ProAir HFA, Teva Pharmaceuticals, North Wales, Pennsylvania). The median mass aerodynamic diameter of the aerosols generated ranges from 2.3 to $3.1 \mu \mathrm{m}$. The jet and vibrating mesh nebulizers have label specifications of 2.7 and $3.1 \mu \mathrm{m}$, respectively, whereas the pMDI has a reported median mass aerodynamic diameter of $2.3 \mu \mathrm{m} .{ }^{19}$

The jet and vibrating mesh nebulizers were filled with albuterol sulfate $(2.5 \mathrm{mg} / 3 \mathrm{~mL}$, Nephron Pharmaceuticals, Orlando, Florida). The jet nebulizer was operated with oxygen at $8 \mathrm{~L} / \mathrm{min}$ using a calibrated flow meter. The unit dose of albuterol sulfate solution was aerosolized by the jet nebulizer until the onset of sputter and by the vibrating mesh nebulizer until no more aerosol was seen.

The pMDI canister, which contained albuterol sulfate (108 $\mu \mathrm{g} /$ actuation), was warmed to hand temperature,

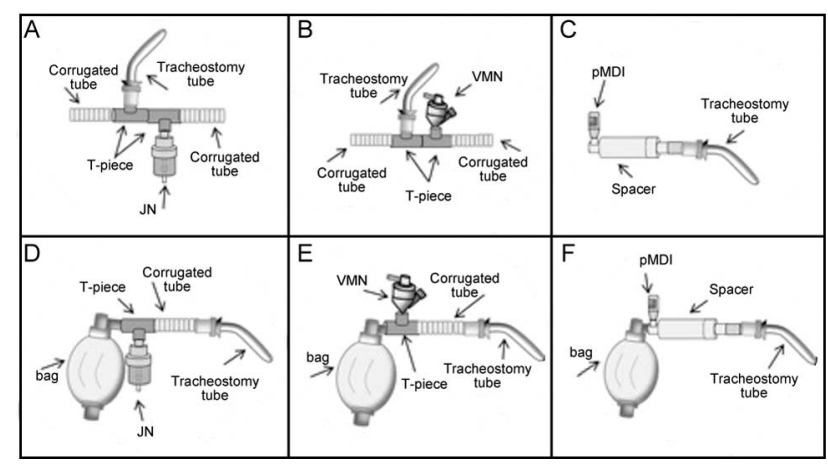

Fig. 2. Experimental setup with each aerosol device using unassisted $(A-C)$ and assisted (D-F) techniques. A: jet nebulizer (JN) with the unassisted technique. B: vibrating mesh nebulizer (VMN) with the unassisted technique. C: pressurized metered-dose inhaler (pMDI) with the unassisted technique. D: jet nebulizer with the assisted technique. E: vibrating mesh nebulizer with the assisted technique. F: pMDI with the assisted technique.

shaken well, and primed with 4 actuations before each experimental run. In each experiment, the pMDI was removed from the actuator, inserted into the nozzle inlet of a spacer (AeroChamber HC MV, Trudell Medical, London, Ontario, Canada), and then actuated at the onset of inspiration for a total of 4 puffs with $>15$ s between actuations. All actuations were performed by the same investigator to minimize interoperator variability.

\section{Administration Techniques}

Aerosol therapy was administered with each device to the simulated spontaneously breathing model by 2 techniques: (1) an assisted technique using a manual resuscitation bag in conjunction with an aerosol device and (2) an unassisted technique with direct administration of aerosols with an aerosol device. All breaths delivered using the manual resuscitation bag were synchronized with each inspiration targeting the same volume and breathing parameters, and they were administered by a single investigator to ensure consistency with the simulated spontaneously breathing model.

Unassisted Technique Setup. The jet and vibrating mesh nebulizers were used as shown in Figure 2 (A and B, respectively). The T-piece of the nebulizers was connected to another T-piece, which was attached to the TT. A 6-inch length of 22-mm inner diameter corrugated tubing was placed on both open ends of the T-pieces. After inserting the pMDI canister into the nozzle inlet of the spacer, it was connected to the TT (Fig. 2C).

Assisted Technique Setup. The jet and vibrating mesh nebulizers were attached to a pediatric manual resuscitation bag with a total volume of $450 \mathrm{~mL}$ (Ambu SPUR II 
Aerosol Delivery in a Pediatric Tracheostomy Model

Table 1. Amount of Albuterol Deposited on a Filter and Calculation of the Percentage of the Drug Dose Placed in the Jet and Vibrating Mesh Nebulizers $(2.5 \mathrm{mg})$ and the Actuated Label Dose Emitted From the pMDI (432 $\mu \mathrm{g})$ Using Both the Assisted and Unassisted Techniques

\begin{tabular}{|c|c|c|c|c|c|c|c|c|}
\hline & \multicolumn{3}{|c|}{ Inhaled Mass (mg) } & \multirow[b]{2}{*}{$P^{*}$} & \multicolumn{3}{|c|}{ Inhaled Mass (\%) } & \multirow[b]{2}{*}{$P \dagger$} \\
\hline & $\begin{array}{c}\text { Jet } \\
\text { Nebulizer }\end{array}$ & $\begin{array}{l}\text { Vibrating Mesh } \\
\text { Nebulizer }\end{array}$ & pMDI & & $\begin{array}{c}\text { Jet } \\
\text { Nebulizer }\end{array}$ & $\begin{array}{l}\text { Vibrating Mesh } \\
\text { Nebulizer }\end{array}$ & pMDI & \\
\hline Assisted technique & $0.13 \pm 0.03$ & $0.43 \pm 0.03$ & $0.18 \pm 0.05$ & .001 & $5.31 \pm 1.59$ & $17.45 \pm 1.26$ & $43.32 \pm 12.38$ & .004 \\
\hline Unassisted technique & $0.15 \pm 0.01$ & $0.49 \pm 0.07$ & $0.20 \pm 0.03$ & .007 & $5.88 \pm 0.77$ & $19.77 \pm 2.99$ & $47.15 \pm 7.82$ & .002 \\
\hline$P \ddagger$ & .46 & .19 & .64 & & .46 & .19 & .64 & \\
\hline \multicolumn{9}{|c|}{$\begin{array}{l}\text { Values are mean } \pm \text { SD. } \\
\text { * Differences among devices in inhaled mass } \\
\dagger \text { Differences among devices in inhaled mass percent } \\
¥ \text { Differences between techniques } \\
\text { pMDI = pressurized metered-dose inhaler }\end{array}$} \\
\hline
\end{tabular}

disposable resuscitator, Ambu, Glen Burnie, MD) via a T-piece adapter that was connected to the TT through a 6-inch length of corrugated tubing (Fig. 2, D and E, respectively). The pMDI and spacer were connected between the manual resuscitation bag and the TT (Fig. 2F).

\section{Data Collection}

Each aerosol device was tested 5 times using both administration techniques. In each trial, the amount of aerosol exiting the TT was captured by an absolute filter. At the end of each trial, the deposited drug was eluted from the filter with $10 \mathrm{~mL}$ of $0.1 \mathrm{~N}$ hydrochloric acid (JT Baker, Phillipsburg, New Jersey) using gentle agitation for $1 \mathrm{~min}$ to ensure proper mixing. The albuterol concentration was then analyzed with spectrophotometry (Beckman Coulter, Fullerton, California) using a quartz cuvette at a wavelength of $276 \mathrm{~nm}$. The spectrophotometer was calibrated before the trials with a holmium oxide filter (Beckman Coulter) to determine wavelength accuracy. It was then set to zero before the next trial by running only the solvent.

\section{Data Analysis}

The amount of drug eluted from the filter was quantified and reported as inhaled mass (IM) and inhaled mass percent (IM\%) of the nominal or emitted dose (mean \pm SD). Nominal dose is used to describe the dose of drug placed in the reservoir of a liquid nebulizer, whereas emitted dose is the mass of dose that leaves the pMDI as aerosol, which serves as the label dose of the drug/device combination. Data analyses were performed using SPSS 18.0 (SPSS, Chicago, Illinois). Repeated-measures analyses of variance $(2 \times 3$ (technique $\times$ device) $)$ were performed to determine the difference in IM and IM\% among the jet nebulizer, vibrating mesh nebulizer, and pMDI. Scheffé post hoc comparisons were done to identify differences between aerosol devices tested in this study using the as- sisted and unassisted techniques. A paired $t$ test was used to compare the assisted and unassisted techniques regarding IM and IM\% for each aerosol device. In all comparisons, significance was defined as $P<.05$.

\section{Results}

Table 1 presents mean \pm SD of albuterol deposited on the filter in milligrams and a calculation of the percentage of the drug dose placed in the jet $(2.5 \mathrm{mg})$ and vibrating mesh $(2.5 \mathrm{mg})$ nebulizers, and the actuated label dose emitted from the pMDI $(432 \mu \mathrm{g})$ using both the assisted and unassisted techniques.

\section{Efficiency of Aerosol Devices and Administration Techniques in Drug Delivery}

Regardless of administration technique, the jet nebulizer had a lower IM and IM\% compared with the vibrating mesh nebulizer ( $P=.002$ for both). In contrast, the pMDI had a higher IM\% than either the jet or vibrating mesh nebulizer $(P=.001$ and $P=.006$, respectively). However, the IM was similar for the pMDI and jet nebulizer $(P=.08)$ but significantly less compared with the vibrating mesh nebulizer $(P=.004$ and $P=.002$, respectively). Although there was a trend toward higher IM and IM\% with the unassisted versus assisted technique, the differences were not significant for the jet nebulizer, vibrating mesh nebulizer, and pMDI (see Table 1).

\section{Discussion}

This is the first study to quantify aerosol delivery via a TT with a spontaneously breathing pediatric model using assisted and unassisted techniques with different aerosol devices. IM\% was significantly greater with the pMDI than with either the vibrating mesh or jet nebulizer when using either the assisted or unassisted technique. This was 
not anticipated, as previous work suggested a similar IM\% with the pMDI and vibrating mesh nebulizer. Nonetheless, IM was greater with the vibrating mesh nebulizer than with either the pMDI or jet nebulizer, due in part to the larger nominal dose compared to the pMDI and the lower residual drug volume compared to the jet nebulizer. Delivery efficiency was similar between the assisted and unassisted techniques regardless of the device used.

\section{Efficiency of Aerosol Devices}

Despite the smaller dose for the pMDI (432 $\mu \mathrm{g})$ compared with the jet nebulizer $(2,500 \mu \mathrm{g})$, the amount of drug delivered distal to the TT was greater with the pMDI than with the jet nebulizer. These findings are consisted with reports by Piccuito and Hess, ${ }^{5}$ who compared aerosol delivery between the jet nebulizer and pMDI using an unassisted technique in a simulated spontaneously breathing adult model with a TT. Although they reported that the pMDI was more efficient than the jet nebulizer in relation to IM\% ( $21 \pm 1 \%$ vs $15 \pm 3 \%, P=.002)$, IM was greater with the jet nebulizer than with the pMDI (382 $\pm 68 \mu \mathrm{g}$ vs $84 \pm 4 \mu \mathrm{g}, P<.001)$. In contrast, we found a lower IM with the jet nebulizer than with the pMDI $(130 \pm 30 \mu \mathrm{g}$ vs $180 \pm 50 \mu \mathrm{g}$ ) using the unassisted technique. Their greater IM obtained with the jet nebulizer may be attributed to the greater fill volume ( $4 \mathrm{~mL}$ vs the drug label of $3 \mathrm{~mL}$ ) used in their study, which led to an increase in aerosol delivery by decreasing the residual volume in the jet nebulizer. ${ }^{20}$ Additionally, they capped one end of the jet nebulizer T-piece, whereas the other end was attached to the T-piece interface via a $15-\mathrm{cm}$ flexible tube, compared with the setup we used in our study (see Fig. 2A). Capping one end of the jet nebulizer T-piece would be expected to direct more of the aerosol into the airway, increasing the amount of delivered medication.

The size of the TT also influences aerosol drug delivery. Pitance et $\mathrm{al}^{6}$ studied the effect of the TT size on aerosol delivery in an adult lung tracheostomy model. They tested different TT sizes $(6.5,8,8.5$, and $10 \mathrm{~mm})$ and found a negative correlation between the TT sizes and aerosol lost in the cannulas. Because removing the inner tracheostomy cannula improved aerosol delivery by up to $31 \%$, they recommended removing the tracheostomy cannula before aerosol therapy in patients with a tracheostomy. ${ }^{6}$ Although differences in aerosol delivery between pediatric and adult TTs have not been reported, it is assumed that aerosol deposition via a pediatric TT is less than with an adult TT.

In this study, we found that the vibrating mesh nebulizer was 3-fold more efficient than the jet nebulizer for both IM\% and IM. This agrees with the findings of Ari et al, ${ }^{21}$ who evaluated aerosol drug delivery with jet and vibrating mesh nebulizers in a mechanically ventilated pediatric lung model with an ETT. However, the IM\% of jet and vibrat- ing mesh nebulizers was marginally lower than that in this study. Previous research led us to expect a 32\% reduction in lung delivery of aerosol with mechanically assisted ventilation versus spontaneous breathing. ${ }^{22}$ It has been reported that the TT is more efficient than the ETT in terms of drug delivery. ${ }^{11}$ In addition, humidity may be an important factor that lowers drug delivery during mechanical ventilation as evidenced in previous studies. ${ }^{5,23}$

\section{Efficiency of Aerosol Administration Techniques}

Although there was a trend toward reduced delivery with the assisted technique, we did not find a significant difference between the assisted and unassisted techniques. In contrast, in an in vitro study of an intubated adult model, Fink et al ${ }^{15}$ reported that when using the same ventilator parameters, the controlled mechanical ventilation mode decreased aerosol delivery from a pMDI by $>30 \%$ compared with simulated spontaneous breathing in CPAP mode. These findings appear to agree with those of the aforementioned study by Dolovich et al. ${ }^{22}$ The result of nonsignificant reduction of drug delivered distal to the TT with the assisted technique in our study could stem from the fact that we used a pediatric lung model with a $\mathrm{V}_{\mathrm{T}}$ and inspiratory flow pattern that closely matched those given by the simulated spontaneously breathing model without adding additional volume or flow from the manual resuscitation bag. These moderate additions of assisted ventilation to the spontaneous breathing pattern were less likely to substantially change the transitional flow and turbulence, both of which can greatly decrease the delivered dose.

Berlinski and $\mathrm{Chavez}^{7}$ reported a lower aerosol delivery with a pMDI using a similar spacer during simulated spontaneous breathing and a greater reduction with the assisted technique. This difference may be due to the lung model developed by Berlinski and Chavez, ${ }^{7}$ which was representative of a 6-y-old child and allowed expiration of the drug. The airway model consisted of a plastic tube with an uncuffed TT inserted and filters on the upper end of the tube (collecting exhaled aerosol) and on the lower end of the tube connected to the breath simulator (collecting inhaled aerosol). The exhaled aerosol would result in a lower level of inhaled dose. In contrast, our model did not allow expiration of the drug and thus resulted in a greater inhaled dose and likely a lower reduction in inhaled dose with assisted ventilation.

In contrast to our findings, both Ari et al, ${ }^{11}$ using an adult tracheostomy model, and Berlinski, ${ }^{8}$ using a spontaneously breathing pediatric model with a tracheostomy, reported an increase in drug delivery using the jet nebulizer with the assisted technique. The discrepancy may be related to use of an adult tracheostomy model with larger volumes by Ari et al, ${ }^{11}$ as larger $\mathrm{V}_{\mathrm{T}}$ and lower breathing 
frequency in adults are associated with greater aerosol delivery. Berlinski ${ }^{8}$ reported that the assisted technique with every other breath delivered more drug than assistance with every breath, and both delivered more drug compared with the unassisted aerosol administration technique with a nebulizer. However, Berlinski ${ }^{8}$ used a different configuration for unassisted delivery (extension tube) and assisted delivery (extension tube with manual resuscitation), with potential difference in reservoir effect between configurations and patterns. In our study, we used the same configuration with both assisted and unassisted delivery, reducing confounding variables.

\section{Clinical Implications}

The clinical efficacy of medical aerosol is largely device- and method-dependent. Therefore, this study provides guidance previously not available. For example, we found the IM\% to be greatest with the pMDI, and 4 puffs delivered a similar amount of drug compared with the jet nebulizer using a larger dose $(2,500 \mu \mathrm{g})$. However, for more expensive formulations and those drugs not available for pMDIs, such as antibiotics, mucokinetics, mucolytics, and prostanoids, the vibrating mesh nebulizer can deliver $>2-3$-fold greater IM than the pMDI and jet nebulizer, making a substantial difference in both cost and effectiveness. Our findings of similar drug delivery between assisted and unassisted aerosol administration techniques with all of the aerosol devices tested suggest that as long as assisted ventilation matches the patient's spontaneous breathing pattern, administration technique selection can be determined by patient comfort level and the need to augment ventilation with a manual resuscitation bag for a specific patient.

Because this was an in vitro study, further clinical research is warranted. We examined only one type and size of TT and resuscitation bag and one set of breathing parameters. In addition, the assisted technique used in this study was designed to closely match the $\mathrm{V}_{\mathrm{T}}$, inspiratory flow, and inspiratory time of the simulated spontaneous breathing patterns. With more aggressive assisted ventilation, a greater reduction in delivered dose might be anticipated.

For future studies, we suggest studying the effect of administration technique on aerosol delivery to simulated pediatric models with a TT by using different breathing patterns, different models simulating severely obstructed airways, and other types of resuscitation bags (such as a flow-inflating bag) with volumes and flows that are blinded to the breath simulator. In the end, clinical studies might be performed to determine whether statistical differences reported in vitro make a clinical difference in infants with severe obstructive airway disease with albuterol or other inhaled drugs.

\section{Conclusions}

Drug delivery distal to the TT was influenced by the type of aerosol device used in this simulated spontaneously breathing pediatric model with a TT. The pMDI was the most efficient in terms of IM\%, whereas the vibrating mesh nebulizer was the most efficient in term of IM due to a larger nominal dose. The jet nebulizer was the least efficient aerosol device for both IM and IM\%. Device selection should be based on the desired delivered dose and the costs associated with achieving that end point. Drug delivery was not significantly different with the assisted and unassisted aerosol administration techniques.

\section{REFERENCES}

1. Dhand R. Special problems in aerosol delivery: artificial airways. Respir Care 2000;45(6):636-645.

2. Dhand R. Basic techniques for aerosol delivery during mechanical ventilation. Respir Care 2004;49(6):611-622.

3. Duarte AG, Fink JB, Dhand R. Inhalation therapy during mechanical ventilation. Respir Care Clin N Am 2001;7(2):233-260, vi.

4. MacIntyre NR. Aerosol delivery through an artificial airway. Respir Care 2002;47(11):1279-1288; discussion 1285-1289.

5. Piccuito CM, Hess DR. Albuterol delivery via tracheostomy tube. Respir Care 2005;50(8):1071-1076.

6. Pitance L, Vecellio L, Delval G, Reychler G, Reychler H, Liistro G. Aerosol delivery through tracheostomy tubes: an in vitro study. J Aerosol Med Pulm Drug Deliv 2013;26(2):76-83.

7. Berlinski A, Chavez A. Albuterol delivery via a metered dose inhaler in a spontaneously breathing pediatric tracheostomy model. Pediatr Pulmonol 2013;48(10):1026-1034.

8. Berlinski A. Nebulized albuterol delivery in a model of spontaneously breathing children with tracheostomy. Respir Care 2013;58(12): 2076-2086

9. Schüepp KG, Straub D, Möller A, Wildhaber JH. Deposition of aerosols in infants and children. J Aerosol Med 2004;17(2):153156.

10. Ari A, Fink JB. Guidelines for aerosol devices in infants, children and adults: which to choose, why and how to achieve effective aerosol therapy. Expert Rev Respir Med 2011;5(4):561-572.

11. Ari A, Harwood RJ, Sheard MM, Fink JB. An in vitro evaluation of aerosol delivery through tracheostomy and endotracheal tubes using different interfaces. Respir Care 2012;57(7):1066-1070.

12. Ari A, Restrepo RD. Aerosol delivery device selection for spontaneously breathing patients: 2012. Respir Care 2012;57(4):613626.

13. Willis LD, Berlinski A. Survey of aerosol delivery techniques to spontaneously breathing tracheostomized children. Respir Care 2012; 57(8):1234-1241.

14. Wyatt ME, Bailey CM, Whiteside JC. Update on paediatric tracheostomy tubes. J Laryngol Otol 1999;113(1):35-40.

15. Fink JB, Dhand R, Duarte AG, Jenne JW, Tobin MJ. Aerosol delivery from a metered-dose inhaler during mechanical ventilation: an in vitro model. Am J Respir Crit Care Med 1996;154(2):382-387.

16. Dolovich MB. Assessing nebulizer performance. Respir Care 2002; 47(11):1290-1301; discussion 1301-1304.

17. Jauernig J, Mitchell J, Berg E, Dennis J, Kreher C, Lamb P, et al. Position paper: recommendation on the adoption of breathing patterns for infants and small children in general chapter 2.9.44. Preparations for nebulisation. Pharmeur Sci Notes 2008;(1):31-32. 


\section{Aerosol Delivery in a Pediatric Tracheostomy Model}

18. Lin HL, Wan GH, Chen YH, Fink JB, Liu WQ, Liu KY. Influence of nebulizer type with different pediatric aerosol masks on drug deposition in a model of a spontaneously breathing small child. Respir Care 2012;57(11):1894-1900.

19. McCabe JC, Koppenhagen F, Blair J, Zeng XM. ProAir HFA delivers warmer, lower-impact, longer-duration plumes containing higher fine particle dose than ventolin HFA. J Aerosol Med Pulm Drug Deliv 2012;25(2):104-109.

20. Hess D, Fisher D, Williams P, Pooler S, Kacmarek RM. Medication nebulizer performance. Effects of diluent volume, nebulizer flow, and nebulizer brand. Chest 1996;110(2):498-505.
21. Ari A, Atalay OT, Harwood R, Sheard MM, Aljamhan EA, Fink JB. Influence of nebulizer type, position, and bias flow on aerosol drug delivery in simulated pediatric and adult lung models during mechanical ventilation. Respir Care 2010;55(7):845-851.

22. Dolovich MB, Killian D, Wolff RK, Obminski G, Newhouse MT. Pulmonary aerosol deposition in chronic bronchitis: intermittent positive pressure breathing versus quiet breathing. Am Rev Respir Dis 1977;115(3):397-402.

23. Miller DD, Amin MM, Palmer LB, Shah AR, Smaldone GC. Aerosol delivery and modern mechanical ventilation: in vitro/in vivo evaluation. Am J Respir Crit Care Med 2003;168(10):1205-1209. 